\title{
STRONGLY REGULAR EXTENSIONS OF RINGS
}

\author{
CARL FAITH ${ }^{11}$
}

As defined by Arens and Kaplansky [2] a ring $A$ is strongly regular (s.r.) in case to each $a \in A$ there corresponds $x=x_{a} \in A$ depending on $a$ such that $a^{2} x=a$. In the present article a ring $A$ is defined to be a s.r. extension of a subring $B$ in case each $a \in A$ satisfies $a^{2} x-a \in B$ with $x=x_{a} \in A$. S.r. rings are, then, s.r. extensions of each subring. A ring $A$ which is a s.r. extension of the center has been called a $\xi$-ring (see Utumi [13], Drazin [3], Martindale [11], and their bibliographies).

Arens and Kaplansky showed that a s.r. ring is a subdirect sum of divison rings. Since any s.r. ring is semisimple, a later result stating that any semisimple $\xi$-ring is a subdirect sum of division rings (see [11]) contains this result. $^{2)}$ In $\$ 2$ of the present article, a further generalization is obtained: (1) If a semisimple ring $A$ is a s.r. extension of a commutative subring $B$, then $A$ is a subdirect sum of division rings. For the proof, the reduction to the case $A$ is primitive is immediate, but at this stage an innovation is made. Instead of specializing $B$, as has been done in the previous work along these lines, a structure theorem (Theorem 2.1) for a primitive s.r. extension $A$ of an arbitrary ring $B$ is obtained first of all: (2) If $A$ is a primitive ring, not a division ring, and if $A / B$ is s.r., then $B$ is dense in the finite topology on $A$. Of course, (1) is an immediate consequence, but more can be squeezed out of (2). For example, (2) shows that in order that a primitive ring $A$ be a s.r. extension of a subring $B$, it is necessary that $B$ be a primitive ring, or an integral domain. (A bit of duality can be introduced here, since in $\S 1$ it is shown that a directly

Received March 22, 1961.

1) This article was written during my tenure as North Atlantic Treaty Organization Postdoctoral Fellow (U.S.A.) in Mathematisches Institut, Heidelberg University, I am presently at the Institute for Advanced Study.

2) A generalization in another direction has been given by Utumi [13]. 
irreducible s.r. extension of an integral domain is necessarily an integral domain.) A fairly easy consequence of this is that in order that a semisimple ring $A$ be a s.r. extension of a subring $B$, it is necessary that $B$ be a subdirect sum of primitive rings and integral domains (Corollary $2.2 \mathrm{ff}$.).

Another consequence of (2) is that any s.r. extension of a division ring is a subdirect sum of division rings. This fact is also implied by the theorem of Arens and Kaplansky inasmuch as a s.r. extension of a s.r. ring is a s.r. ring. However, this and the above results are all obtained in a new way independently of the previous results for $\xi$-rings and s.r. rings.

The structure of $A$ is not known in the general case when $A$ is a s.r. extension of a commutative subring $B$. However the centralizer of $B$ in $A$ is a $\xi$-ring, so that some information on the structure of $A$ is available.

In $\$ 3$ the results on s.r. extensions are applied in extending the results of Nakayama [12] on the commutativity of rings, continuing a program which I began in [4]. Any future improvements in the theory of s.r. extensions will net corresponding improvements in this direction also.

A simple computation shows that a ring is regular $(a x a=a)$ in the sense of von Neumann if and only if every principal one-sided ideal has an idempotent generator. Arens and Kaplansky introduced the notion of strong regularity $\left(a^{2} x=a\right)$, whereby not only are these idempotent generators demanded but also nilpotent elements are banished. Here, and more generally in $\xi$-rings, the emphasis has shifted from the manufacture of idempotents to the disposition of the nilpotent elements of index two: they must all lie in the center. In $\S 5$ the position in a primitive ring $A$ of the subring $T(A)$ generated by the nilpotent elements of index two is investigated. One finds in important special cases (e.g., if $A$ is an algebraic algebra, or if $A$ has a minimal left ideal) that the subring $T(A)$, and also the subring $E(A)$ generated by the idempotents of $A$, is dense in $A$, if $A$ is not division. This clearly illustrates my allusion above to the extent to which the structure of an s.r. extension $A_{/}^{\prime} B$ is influenced by the fact that $B$ contains the subring $T(A)$.

1. Directly jrreducible strongly regular extensious. If $A$ is a $\hat{\xi}$-ring with center $Z$, and if $a, x \in A$ satisfy $a^{2} x-a \in Z$ then [11, Theorem 1] states that $a x=x a$. The verbatim proof (accredited to Herstein) given there establishes 
this implication for $C N$-rings ${ }^{3)}$, a fact which is stated in the proposition below. By reproducing its proof here, I have been able to make this section, and the section following, relatively self-contained.

Proposition 1.1. In any $C N$-ring, any two elements $a$ and $x$ which satisfy $a^{2} x-a \in Z$ are commutative, that is, then $a x=x a$.

Proof. Since $a^{2} x-a \in Z,\left(a^{2} x-a\right) a=a\left(a^{2} x-a\right)$, and so (1) $a^{2}(a x-x a)=0$. Using (1), it follows that $[a(a x-x a) a]^{2}=0$, so that, since $A$ is $C N$, (2) $a(a x-x a) a \in Z$. Commuting this with $a$ and using (1), there results (3) $a(a x-x a) a^{2}=0$. Since $a^{2} x-a \in Z$, one easily verifies that

(4) $a x-x a=[a(a x-x a)+(a x-x a) a] x$.

If (4) is multiplied on the left by $a$, using (1), the result can be simplified to $a(a x-x a)=a(a x-x a) a x$, so that, by (2), one has

$$
\text { (5) } a(a x-x a)=a(a x-x a) a x=x a(a x-x a) a \text {. }
$$

Multiplying (5) on the right by $a$ produces (6) $a(a x-x a) a=x a(a x-x a) a^{2}$, which is $=0$ by (3). Reapplying this latter fact to (5) yields (7) $a(a x-x a)$ $=0$. Thus, (4) can be simplified to (8) $a x-x a=(a x-x a) a x$. From (7) $[(a x-x a) a]^{2}=0$, so that $(a x-x a) a \in Z$. Commuting this with $a$, and using (7), one obtains (9) $(a x-x a) a^{2}=0$. Since $(a x-x a) a \in Z$, (8) becomes $(a x-x a)$ $=x(a x-x a) a$, and so, by (9), one has (10) $(a x-x a) a x=x(a x-x a) a^{2} x=0$. Then (8) reduces to $a x=x a$, which is the desired result.

Corollary 1.2. If $a$ and $x$ are elements of a ring $A$, and if the element $a^{2} x-a$ and all nilpotent elements of $A$ commute with both $a$ and $x$, then $a$ and $x$ commute.

Proof. Let $Q$ denote the subring of $A$ generated by $a$ and $x$, and let $z$ denote the center of $Q$. Then the condition of the corollary implies that $Q$ is a $C N$-ring, and that $a^{2} x-a \in 3$, so that the corollary follows from the proposition.

An element $a$ of a ring $A$ is (von Neumann) regular if $a x a=a$, and strongly

3) A $C N$-ring is a ring in which every nilpotent element belongs to the center. It seems that in each case where I assume that a ring is $C N$, I actually require only that the center contains all nilpotent elements of index two. I do not know whether this latter condition is equivalent to the $C N$ hypothesis. 
regular (Arens and Kaplansky) if $a^{2} x=a$, for suitable $x \in A$. The next corollary in the case $n=1$ shows that in $C N$-rings strong regularity of an element $a$ implies its regularity. The corollary follows from the proposition by observing that $a^{n+1} x=a^{n}$ implies $\left(a^{n}\right)^{2} x^{n}=a^{n}$.

Corollary 1.3. If $A$ is a $C N$-ring, then the equation $a^{n+1} x=a^{n}$ for two elements $a$ and $x$ in $A$, and a natural number $n$, implies the equation $a^{n} x^{n}$ $=x^{n} a^{n}$.

Lemma 1.4. A directly irreducible $C N$-ring $A$ has an identity element $1 \neq 0$ if and only if there exist $a, x \in A$ such that $a^{2} x=a \neq 0$. Then $a x=x a=1$.

Proof. By the corollary $a^{2} x=a$ implies $a x=x a$, so that $e=a x$ is a nonzero idempotent when $a \neq 0$. Since the sets $e A(1-e),(1-e) A e$ are central, they commute with $e$, whereby they are $=0$. By the direct irreducibility of $A, e=1$. The converse is trivial.

In a s.r. extension of a division ring, to each element $a$ there corresponds an element $x$ such that $a^{2} x-a$ has certain regularity properties. This situation for directly irreducible rings is slightly generalized directly below, and following this a similar generalization of s.r. extensions of integral domains is considered.

TheOREM 1.5. Let $A$ be a directly irreducible $C N$-ring containing a left identity $1 \neq 0$, and such that to each $a \in A$ there correspond $b \in A$ and a natural number $n=n_{a}$ such that either $a^{n+1} b-a^{n}=0$, or else $a^{n+1} b-a^{n}$ has a right inverse in $A$. Then the totality $N$ of nilpotent elements of $A$ is an ideal, and $A-N$ is a division ring.

Proof. By Lemma 1.4, 1 is a two-sided identity. If $a^{n+1} b-a^{n}$ has the right inverse $x$, then $a$ has the right inverse $a^{n} b x-a^{n-1} x$. If $a^{n+1} b=a^{n}$, then, by Corollary $1.3, a^{n} b^{n}=b^{n} a^{n}$, so that $e=a^{n} b^{n}$ satisfies $e^{2}=e$. Then, by Lemma 1. 4, either $e=1$, whence $a$ has right inverse $a^{n-1} b^{n}$, or else $0=e=e a^{n}=a^{n}$. Thus, every nonnilpotent element has a right inverse. It is easy to see that this means that every nonnilpotent element has a two-sided inverse. Then (e.g., [9, p. 21]), since $N$ is a (central) ideal of $A, A-N$ is division.

Remark. One can show in general that in a ring $A$ with identity such that every nonnilpotent elemen' has an inverse, that $N$ is a two-sided ideal such 
that $A-N$ is division (cf. the proof of Lemma 1.9 below.)

Corollary 1.6. If $A$ is directly irreducible with a left identity $1 \neq 0$ such that to each $a \in A$ there corresponds $b \in A$ such that either $a^{2} b-a=0$, or else $a^{2} b-a$ has a right inverse, then $A$ is division.

Proof. It is trivial to show that $N=\mathbf{0}$, so that $A$ is $C N$, and the theorem applies.

The corollary shows that a nonzero directly irreducible s.r. ring is division if only if there exists a left identity. In such a ring a two-sided identity exists, according to Lemma 1.4 .

COROLlary 1.7. A nonzero directly irreducible ring is s.r. if and only if it is division.

Theorem 1.8. If $A$ is a directly irreducible $C N$-ring, and if to each $a \in A$ there correspond $b \in A$ and a natural number $n=n_{a}$ such that $a^{n+1} b-a^{n}$ is not a proper right divisor of zero in $A$, then the set $N$ of nilpotent elements of $A$ is a nil ideal, and $A-N$ is an integral domain.

Proof. In a way completely analogous to the proof of the last theorem, one sees that $N$ coincides with the set $D$ of all right divisors of 0 in $A$. Thus, the theorem is a consequence of the following lemma. The lemma no doubt is known, but I have not been able to find a published proof. For this reason, I include one here.

Lemma 1.9. If $N=D$ in a ring $A$, then $N$ is an ideal of $A$, and $A-N$ is an integral domain.

Proof. If $N=\mathbf{0}$, there is nothing to prove. Now let $0 \neq x \in N$ have index of nilpotency $=m$. Then, since $(a x) x^{m-1}=0, a x \in D=N$, for all $a \in A$, that is, $A x \subseteq N$, for all $x \in N$. Since $(a x)^{n}=0$ implies that $(x a)^{n+1}=0$, this shows that $A x \subseteq N$ implies that $x A \subseteq N$, so that $A x A \subseteq N$, for all $x \in N$. In order to show that $N$ is an ideal, it. renains to show that $N$ is closed under addition. If $x, y \in N$, then, since $(x+y)^{3} \in A x A+A y A$, it follows that $(x+y) \in N$. It remains to show that $A-N$ is integral. It suffices to show that $a \notin N, b \notin N$, $a b=q \in N$ leads to a contradiction. Clearly, $q \neq 0$, so $q$ is nilpotent of index $m \geqq 2$. Since $(a b)^{m}=\left[(a b)^{m-1} a\right] b=0, b \notin D$ implies that $(a b)^{m-1} a=0$. But $a \notin D$ implies that $(a b)^{m-1}=0$, which is the desired contradiction. 
A consequence of Corollary 1.6 is that a directly irreducible s.r. extension of a division ring is a division ring. In analogy with this fact one has

CoRollary 1.10. A directly irreducible s.r. extension of an integral domain is an integral domain.

Proof. If $A$ is the extension, and $B$ the integral domain, then $A$ contains no nilpotent elements $\neq 0$. Thus, if $a, y \in A$, then $a y=0$ if and only if $y a=0$. Since $A$ is a $C N$-ring, by the theorem it suffices to show that $a-a^{2} b \in B$ is not a proper right divisor of zero in $A$. Hence assume that $0 \neq y \in A$ is such that $y\left(a-a^{2} b\right)=0$, and $a-a^{2} b \neq 0$. Then $\left(a-a^{2} b\right) y=0$, so that $\left(a-a^{2} b\right)\left(y-y^{2} c\right)=0$, where $c$ can be chosen such that $y-y^{2} c \in B$. Since $B$ is integral, $y=y^{2} c$, so that, by Lemma $1.4, y c=c y$ is the ring identity, which contradicts the choice of $y$ as a proper left divisor of 0 .

Since a s.r. ring is a s.r. extension of every subring, it would seem that the hypothesis " $A$ is a s.r. extension of $B$ " would have more force if one assumes at the outset that $A$ is not an s.r. ring. (Then $B$ is not s.r.!) For these rings the structure theory can be reduced in some cases to that of directly irreducible s.r. extensions.

Proposition 1.11. Let $B$ be a simple ring with identity $e$, and let $A$ be $a$ s.r. extension of $B, A$ not a s.r. ring. Then $A=Q \oplus P$, where $Q$ is a directly irreducible s.r. extension of $B$ having the identity $e$, and $P$ is a s.r. ring. Conversely, $Q \oplus R$ is a s.r. extension of $B$, if $Q$ is any s.r. extension of $B$, and $P$ is any s.r. ring.

Proof. The sufficiency is clear. The necessity requires the following lemma which is also of interest in more general situations.

LeMma. If $B$ is a ring with a central idempotent $e$, and if $A / B$ is $a$ s.r. extension, then $e$ is a central element of $A$.

Proof of the Lemma. $B$ contains all nilpotent elements of index two, so that $B$ contains the sets $e A(1-e),(1-e) A e$. Since $e$ is central in $B$, these sets $=0$, so $A=e A e \oplus(1-e) A(1-e)$, and $e$ is central.

Going back to the proof of the proposition, since $(1-e) A(1-e) \cap B=0$, $P=(1-e) A(1-e)$ is s.r. as required. It remains to show that $Q=e A e$ is directly irreducible. To this end assume that $Q=M \oplus N$, where $M$ and $N$ are 
ideals. $\quad M \cap B=0$ implies that $M$ is s.r. If both $M$ and $N$ were s.r., then so would $Q$, whence $A$, be s.r., contrary to assumption. On the other hand, if $M \cap B \neq 0$, then $M \supseteqq B$, so that $M=Q, N=\mathbf{0}$, and $Q$ is directly irreducible.

The existence of directly irreducible s.r. proper extensions, not division rings, of simple rings is guaranteed by the example in $\S 4$ of [4].

§2. Semisimple strongly regular extensions. The next theorem shows that in order that a ring $B$ possess a primitive s.r. extension, it is necessary that $B$ be a primitive ring, or an integral domain.

2.1. Structure Theorem. Let $A$ be a primitive ring, not a division ring, which is represented as a dense ring of l.t.'s in a vector space $V$ over a division ring $D$. Then: if $B$ is any subring of $A$ such that $A / B$ is s.r., then $B$ is isomorphic to a dense ring of l.t.'s in $V$.

Proof. Let $V_{n}$ be a vector subspace of $V$ of finite dimension $n$, let $U=\left\{a \in A \mid V_{n} a \subseteq V_{n}\right\}$, and let $K=\left\{a \in A \mid V_{n} a=\mathbf{0}\right\}$. Then, as is well known [9], the difference ring $\bar{U}=U-K$ is isomorphic to $D_{n}$, the complete ring of $n \times n$ matrices over $D$. First assume that $n>1$, and let $u \in U$ be such that $u^{2} \in K$. Then, if $c \in A$ is such that $u-u^{2} c \in B$, then, since $u^{2} c \in K$, it follows that $u-u^{2} c \in Q=B \cap U$. Thus, the subring $\bar{Q}$ determined by $Q$ under the canonical homomorphism $U \rightarrow \bar{U}$ contains every $\bar{u} \in \bar{U}$ satisfying $\bar{u}^{2}=0$. By $[7$, p. 602 , Proposition 1], $\bar{Q}=\bar{U}$, that is, $U=Q+K$, and, consequently, every l.t. of $V_{n}$ is induced by an element of $B$, in case $n>1$. Now $V_{1}$ is contained in a subspace $V_{2}$, and if $\bar{a}_{1}$ is any 1.t. in $V_{1}$, there exists a 1.t. $\bar{a}_{2}$ in $V_{2}$ such that $a_{2}$ induces $\bar{a}_{1}$. Then, if $b \in B$ induces $\bar{a}_{2}$, then $b$ also induces $\bar{a}_{1}$. Thus, in all cases, the 1.t.'s in $V_{n}$ can be induced by elements of $B$. This establishes that $B$ is isomorphic to a dense ring of 1.t.'s in $V$.

(1) of the next corollary is immediate.

Corollary 2.2. Let $A$ be a s.r. extension of a ring $B$. (1) If $A$ is a primitive ring, not a divison ring, then $B$ is a primitive ring, and so is any intermediate ring of $A / B$. (2) If $A$ is semisimple, then $B$ is a subdirect sum of primitive rings, and integral domains.

Proof. (2) Let $\{P\}$ denote the collection of primitive ideals in $A$. Since 
$\cap P=0$, necessarily $\cap(P \cap B)=0$, so that $B$ is a subdirect sum of the rings $\{B-(P \cap B)\}$. Now $A-P$ is a s.r. extension of $(P+B)-P$, so that by the theorem: if $A-P$ is not a division ring, then $(P+B)-P$ is primitive; $(P+B)-P$ is an integral domain, otherwise. (2) is completed by observing that $(P+B)-P$ is isomorphic to $B-(P \cap B)$, for each $P \in\{P\}$.

Corollary 2.3. Let $A$ be a semisimple ring which is a s.r. extension of $a$ commutative subring $B$. Then $A$ is a subdirect sum of division rings, and $B$ is a subdirect sum of (commutative) integral domains. (If in addition $A$ is subdirectly irreducible, then $A$ is a division ring).

Proof. Let $A^{\prime}$ be any primitive homomorph of $A$, and let $B^{\prime}$ denote the corresponding map of $B$. Since $A^{\prime} / B^{\prime}$ is s.r., and $B^{\prime}$ is commutative, density of $B^{\prime}$ in $A^{\prime}$ would imply commutativity of $A^{\prime}$, which in turn would imply that $A^{\prime}$ is a field. Thus, by the theorem, $A^{\prime}$ is a division ring, so that $A$ is a subdirect sum of division rings. By the corollary, $B$ must be a subdirect sum of (commutative) primitive rings and integral domains. Since a commutative primitive ring is a field, $B$ has the desired struture. (The parenthetical remark is obvious).

3. Commutativity theorems. If $S$ is a nonempty subset of a ring $A$, then [S] denotes the subring generated by $S$. If $R$ is a subring, then $R[S]$ denotes the subring generated by $R$ and $S$. If $A$ is a division ring, and if $R$ is a division subring, $R(S)$ is the division subring generated by $R$ and $S$.

Let $\Phi$ be a commutative ring with identity. A ring $A$ is a $\emptyset$-ring (in the sense of Jacobson [8, p. 55]) if $A$ is a unitary left $\Phi$-module satisfying $c(x y)=(c x) y=x(c y)$ for all $c \in \emptyset$, and all $x, y \in A$.

Definition. Let $\Phi$ be a commutative ring with identity which contains a (possibly 0) subring $K$ with the property that (1) a nonzero homomorph $K^{\prime}$ of $K$ is an integral domain if and only if $K^{\prime}$ is an algebraically closed field, and (2) there exist finitely many $c_{1}, \ldots, c_{r} \in \emptyset$ such that $\Phi=K\left[c_{1}, \ldots, c_{r}\right]$. Let $A$ be a $\Phi$-ring, and $B$ a $\Phi$-subring of $A$ such that to each $a \in A$ there corresponds a polynomial $P_{a}(x)$ in the polynomial ring $\mathscr{D}[x]$ such that

$$
a^{n}-a^{n+1} p_{a}(a) \in B
$$

for some natural number $n$ depending on $a$. Then $A / B$ is an $N$-extension. If 
$A / B$ is an $N$-extension, then it is an $N_{1}$-extension if $n=1$ for all $a \in A$, and it is an $N_{2}$-extension if $B$ contains all idempotents of $A$.

$N$-extensions have been studied extensively by Nakayama [12] (and others, see [12, References]) where the main result states that any $\operatorname{ring} A$ which is an $N_{1}$-extension of its center $Z$ is commutative (or, more generally, any $C N$ ring which is an $N$-extension of its center is commutative.) This result had been obtained earlier by Nakayama in the case $K=\mathbf{0}$. In this case it is also true that a division ring $A$ is commutative if it is an $N$-extension of a division $\Phi$-subring $\neq A$ (Faith [4, Theorem 1]), a result which is extended to the $K \neq \mathbf{0}$ case below.

Theorem 3.1. Let $A$ be a division $\emptyset$-ring, $\Phi$ as in the definition, and let $B$ be a $\emptyset$-subring such that $A / B$ is an $N$-extension. Then: if $B$ is commutative, or if $B$ is a division subring $\neq A$, then $A$ is a field.

Proof. If $B$ is commutative, so is the division subring $(B)$ generated by $B$. If $(B)=A$, then $A$ is a field as required. Hence, it suffices to consider only the case where $A(\neq Z)$ is an $N$-extension of the division ring $B \neq A$. Let 1 be the identity of $A$, and set $\varphi=\emptyset 1$. Since $\varphi \neq 0, A$ and $B$ are algebras over the field $\bar{\varphi}$ of quotients of $\varphi$. In this case the results of [6] are applicable. The hypotheses imply that to each $a \in A$ there corresponds $p_{a}(x)$ with coefficients in $\varphi(\subseteq \bar{\varphi})$ such that $a^{n}-a^{n+1} p_{a}(a) \in B$, Under these conditions [6, Theorem 1.5] asserts that to each $b \in A$ there corresponds a polynomial $F_{b}(x)$ over $\bar{\varphi}$ such that (i) $F_{b}(b) \in Z$, and (ii) $F_{b}(x)$ is the composition of finitely many of the polynomials in the set

$$
\left\{x^{n}-x^{n+1} p_{a}(x) \mid a \in A, n=1,2, \ldots\right\} .
$$

Clearly, then, the polynomial $F_{b}(x)$ has the form

$$
F_{b}(x)=x^{m}-x^{m+1} g_{b}(x),
$$

with $m=m(b)>1$, and $g_{b}(x) \in \varphi[x]$. (It is important to note that the $F_{b}(x)$ are polynomials over $\varphi$.) The effect of all of this is to show that $A / Z$ is an $N$-extension, as defined above, so that $A=Z$ by the result of Nakayama.

Theorem 3.2. Let $A$ be a $\emptyset$-ring, $\Phi$ as in the definition, and let $B$ be a commutative 0 -subring such that $A / B$ is an $N_{1}$-extension. If either $A$ is semi- 
simple, or $B \cap J(A)=0$, where $J(A)$ denotes the Jacobson radical of $A$, then $A$ is commutative.

Proof. Since $A$ is semisimple (if $B \cap J(A)=\mathbf{0}$, then $J$ is s.r., so $J=0$ ), $A$ is a subdirect sum of division rings $A^{\prime}$ by Corollary 2.3. Each $A^{\prime}$ can be regarded as a $\emptyset$-ring, and it follows that each $A^{\prime}$ is an $N_{1}$-extension of a commutative subring, so that each $A^{\prime}$ is commutative by Theorem 3.1. Then $A$ is commutative.

Below, if a ring is an $N_{1}$-extension of $\mathbf{0}$, then it is an $N_{1}$-ring. By Nakayama's result, every $N_{1}$-ring is commutative. If $A$ is an $N_{1}$-extension of a simple subring $B$, and if $B$ has an identity $e$, it follows from the lemma to Proposition 1.11 that $A=e A e \oplus(1-e) A(1-e)$. Since $(1-e) A(1-e)$ is an $N_{1}$-ring, it is commutative. Now suppose that $e A e=M \oplus N$, where $M$ and $N$ are ideals. If both $M$ and $N$ are disjoint from $B$, then both $M$ and $N$ are $N_{1}$. rings, whence they are commutative. Thus, if $e A e$ is noncommutative, it can be assumed that, say, $B \cap M \neq 0$. Then, by the simplicity of $B, B \cong M$, and, since $M$ now contains the identity $e$ of $e A e, M=e A e, N=0$, so that $e A e$ is directly irreducible. This establishes the lemma.

Lemma 3.3. If $A$ is an $N_{1}$-extension of a simple $\emptyset$-subring $B$, and if $B$ contains an identity element $e$, then

$$
A=Q \oplus P,
$$

where $Q=e A e$, and $P=(1-e) A(1-e)$ is a (commutative) $N_{1}$-ring. Furthermore, either $A$ is commutative, or else $e A e=Q$ is directly irreducible.

Now suppose that $B$ in the lemma is a division $\Phi$-subring. Then, if $A$ is noncommutative, $Q$ is a directly irreducible $N_{1}$-extension of $B$. Since Corollary 1.7 shows that $Q$ is a division ring, it follows from Theorem 3.1 that either $B=Q$, or else $Q$ is a field. This completes the proof of the next theorem.

TheOREM 3.4. Let $A$ be $a$ $\emptyset$-ring, and $B$ a division $\emptyset$-subring such that $A / B$ is an $N_{1}$ extension. Then, either $A$ is commutative, or else $A=B \oplus P$, where $P$ is a (commutative) $N_{1}$-ring. Furthermore, if $A$ is directly irreducible, and $B \neq A$, then $A$ is a field.

The theorem and the discussion preceding have the corollary. 
Corollary 3.5. If $A$ is a $\Phi$-ring which is an $N_{1}$-extension of a $\emptyset$-subfield $B$, then $A$ is commutative.

$\S 4$. $\xi_{2}$-extensions. The extension $A / B$ is a $\xi$-extension in case to each $a \in A$ there exist $x=x_{x} \in A$ and a natural number $n=n_{x}$ such that $a^{n}-a^{n+1} x \in B$. If $x$ can be chosen such that $x^{n}$ commutes with $a^{n}$, for every $a \in A$, then a $\xi$-extension is a $\xi^{\prime}$-extension. A $\xi$-extension is $\xi_{2}$, if $B$ contains all idempotents of $A$, and $\xi_{2}^{\prime}$ if it is both $\xi_{2}$ and $\xi^{\prime}$.

If $A$ is a $D$-ring, where $\mathscr{D}$ is a commutative ring with identity, and if $B$ is a $\mathscr{D}$-subring such that to each $a \in A$ there correspond $p_{a}(x) \in \Phi[x]$ and a natural number $n=n_{a}$ such that $a^{n}-a^{n+1} p_{a}(a) \in B$, then $A / B$ is a $\xi^{\prime}$-extension ; it is $\xi_{2}^{\prime}$ if $p_{e}(e)=0$ for each idempotent $e \in A$. Thus, the results of this section are applicable to these extensions; in particular, they are applicable to $N$ extensions.

A ring $A$ is a $\xi_{2}^{\prime}$-ring if it is a $\xi_{2}^{\prime}$-extension of $\mathbf{0}$. It is trivial to verify that any $\xi_{2}^{\prime}$-ring is a nil ring, and conversely. If $A / B$ is $\xi_{2}^{\prime}$, and if $L$ is any left ideal disjoint from $B$, then $L$ is nil. To see this, if $a \in L$, and if $a^{n}-a^{n+1} x$ $\in B$, then $0=a^{n}-a^{n+1} x \in B \cap L=0$. Since $a^{n} x^{n}=x^{n} a^{n}$, this implies that $e=a^{n} x^{n}$ is idempotent. Since $e \in L \cap B=0$, then $a^{n}=e a^{n}=e=0$, so that $L$ is nil. This fact is used several times below.

THEOREM 4.1. If $A$ is a $\hat{5}_{2}^{\prime}$-extension of a simple ring $B$, and if $J(A) \neq A$, then $J(A)$ is nil, and $A-J(A)$ is primitive.

Proof: Suppose for the moment that $J(A) \supseteqq B$. Then $A-J(A)$ would be a $\xi_{2}^{\prime}$-ring, whence it is a nil ring. This would imply that $A=J(A)$, which is excluded by hypothesis. Hence $J(A) \neq \equiv$, so that $J(A) \cap B=\mathbf{0}$, whence $J(A)$ is nil. Now $B$ cannot be contained in every primitive ideal of $A$, since the intersection of these is $J(A)$. Hence there exists a primitive ideal $P$ which is disjoint from $B$. Then $P$ is nil, whence $\left.P=J^{\prime} A\right)$, and $A-J(A)$ is primitive.

Now suppose that $A$ is a ring with no nil ideals $\neq 0$ which is a $\xi_{2}^{\prime}$-extension of a division subring $B$. By the theorem, $A$ is primitive, but, as a matter of fact, $A$ is division. The proof of this is similar to the proof of the theorem, except that one considers the modular maximal left ideals (m.m.l.-ideals) of $A$ instead of the primitive ideals. Since $A$ contains no nil left ideals, one concludes that $\mathbf{0}$ is a m.m.l.-ideal, that is, that $A$ is a division ring. This fact is stated 
in the next theorem.

Theorem 4.2. If $A$ is a ring with no nil ideals $\neq 0$, and if $A$ is $a \xi_{2}^{\prime}$ extension of a division subring, then $A$ is a division ring.

The corollary below is a consequence of the theorem, and of Theorem 3.1.

Corollary 4.3. If $A$ is a ring with no nil ideals $\neq 0$, and if $A$ is a $N_{2}$ extension of a division subring $B \neq A$, then $A$ is a field.

The last two results can be restated as follows: If $A$ is a ring containing no nonzero idempotents $\neq 1$, and containing no nonzero nil ideals, and if $A$ is a $\xi^{\prime}$-extension (resp. $N$-extension) of a division subring $B \neq A$, then $A$ is a division ring (resp. field.)

The corollary generalizes results on radical extensions of [4] and [5].

If $A$ is a radical extension of an integral domain, then to each $a \in A$ there corresponds a natural number $n$ such that $a^{n}$ has certain regularity properties. The situation is generalized below.

Theorem 4.4. Let $A$ be a ring wiih the property that to each $a \in A$ there corresponds a natural number $n=n_{a}$ such that $a^{n}$ is not a proper right divisor of zero in $A$. Then the set $N$ of nilpotent elements is an ideal, and $A-N$ is an integral domain.

Proof. Let $D$ denote the set of all right divisors of zero in $A$. The condition of the theorem implies that $N=D$, so that the theorem follows from Lemma 1.9.

Remark. If $A$ is a ring with a nil ideal $N$ such that $A-N$ is integral, then, of course, $D=N$ in $A$, and $A$ has the property of the theorem.

Now let $A$ be a radical extension of an integral domain $B$, that is, such that to each $a \in A$ there corresponds a natural number $n=n_{a}$ such that $a^{n} \in B$. Assume that $A$ contains no nil left ideals $\neq 0$, let $x \in A$ be nonnilpotent, and let $y \in L_{x}=\{a \in A \mid a x=0\}$. Then, since $y^{m} x^{n}=0, m=m_{y} . \quad n=n_{x}$, since $B$ is integral, and since $x^{n} \neq 0$, then $y^{m}=0 . \quad L_{x}$ is therefore nil, so $L_{x}=0$. This shows that each $a \in A$ has the property stated in the theorem, and completes the proof of the corollary.

Corollary 4.5. If $A$ is a ring with no nil lest ideals $\neq 0$, and if $A$ is a radical extension of an integral domain, then $A$ is an integral domain. 
A commutative integral domain $A$ can be radical over a subring $B$ only under very special circumstances. For then, if $A^{*}$ and $B^{*}$ denote the respective quotient fields of $A$ and $B$, then $A^{*}$ is radical over $B^{*}$. It follows from the work of Kaplansky [Canad. J. Math. vol. 3 (1951) 290-292] that either $A^{*}=B^{*}$, or else, $A^{*}$ has characteristic $p>0$, and either $A^{*} / B^{*}$ is purely inseparable, or else $A^{*}$ is algebraic over $G F(p)$. It would be interesting to know the corresponding situation for noncommutative integral domains (cf. [5] for some results with added hypotheses on $A$ and $B$ ).

5. Generation of primitive rings. If $A$ is a ring, let $T(A)$ denote the subring generated by all nilpotent elements of index two, and let $E(A)$ be the subring generated by all idempotents. If $A$ is primitive, and $A / B$ is s.r., then by Corollary $2.2, B$ is dense in the finite topology on $A$, if $A$ is not division. In view of the fact that $B$ contains $T(A)$ when $A / B$ is s.r., it would be interesting to know if any subring of $A$ which contains $0 \neq T(A)$ is dense in $A$. Positive results abound in special cases, making a counterexample hard to find.

THEOREM 5.1. If $A$ is a primitive ring with a minimal left ideal, and if $A$ is not a division ring, then $T(A)$ and $E(A)$ are dense in the finite topology on $A$. ' (Then $T(A)$ and $E(A)$ are primitive rings).

Let $S$ denote the socle of $A$. It suffices to show that $T(S)=E(S)=S$, since then density follows from the inclusions $T(A) \supseteqq S, E(A) \supseteqq S$. Thus, the theorem is a consequence of the lemma below. (In case $A$ does not satisfy the minimum condition, then the theorem follows immediately from Rosenberg's generalization [Proc. Amer. Math. Soc. vol. 7 (1956) p. 897, Corollary 5] of a theorem of Kasch [10]).

LEMMA 5.2. (a) If $A$ is a simple ring containing a nontrivial idempotent, then $T(A)=A$. If, in addition, (b) $A$ is an algebra over a field $\Phi \neq G F(2)$, or (c) if A contains a minimal left ideal, then $E(A)=A$.

Proof. (a) Let $\mathscr{T}$ denote the additive subgroup generated by all nilpotent elements of index two, and let, for any subset $S$ of $A,[S, S]$ denote the additive subgroup generated by all $[a, b]=a b-b a, a, b \in S$. If $u, v \in \mathscr{T}$ are nilpotent of index two, then so is

$$
w=(1+u) v(1-u)
$$


Then,

$$
[u, v]=w+u v u-v \in \mathscr{T} .
$$

It easily follows from this that $\mathscr{T}$ is a Lie ring with respect to $[a, b]$. Then Amitsur's [1, Lemma 2] shows that $\mathscr{T} \supseteqq[A, A]$, so that $T(A) \supseteqq[A, A]$. If $e$ is any nontrivial idempotent in $A$, then $e A f, f A e \subseteq \mathscr{T} \leqq T(A)$, where (formally) $f=1-e$. But $T(A)$ also contains the product

$$
e A e=e(A f A) e=(e A f)(f A e)
$$

similarly, $f A f \subseteq T(A)$. Then $T(A)=A=e A e+e A f+f A e+f A f$, as needed.

(b) In this case Amitsur's [1, Theorem 1] states that $A$ contains no noninvariant noncentral subalgebras $\neq A$, unless $A$ is 4-dimensional over a field $F$ of characteristic two. Since $E(A), T(A)$ are invariant noncentral subalgebras, equality $E(A)=T(A)=A$ follows when $\operatorname{dim} A / F \neq 4$. In this exceptional case, $A$ is a simple matrix algebra. A general property of arbitrary matrix algebras $A=R_{n}, n>1$, implied by [7, Prop. 1] is that $E(A)=T(A)=A$. This latter result also suffices for the case (c), since $A$ is then locally a complete matrix ring $R_{n}, n>1$, by Litoff's theorem [8, p. 90].

THEOREM 5.3. Let $A$ be an algebraic algebra over the field $\emptyset$. (a) If $A$ is primitive, but not division, then $E(A)$ and $T(A)$ are dense in the finite topology on $A$. (Then $E(A)$ and $T(A)$ are primitive algebras.) (b) If $A$ is semisimple, so is $E(A)$.

Proof. (a) The proof is analogous to that of Theorem 2.1. Adopting the terminology there, with $B=E(A)$ (resp. $B=T(A)$ ), if $\bar{e}$ is any element in a complete set of matrix units for $\bar{U}$, by [9, p. $239 \mathrm{ff}$.$] , there exists an element$ $f$ in a complete set of matrix units in $U$ such that $\bar{f}=\bar{e}$. If $\bar{e}^{2}=\bar{e}\left(\right.$ resp. $\left.\bar{e}^{2}=0\right)$, then, since $f \in E(A)$ (resp. $f \in T(A)$ ), it follows that $\bar{e} \in \bar{Q}$. Since any automorphism of $\bar{U}$ maps a complete set of matrix units onto another complete set, this latter assertion shows that $\bar{Q}$ contains all conjugates of $\bar{e}$. Since $\bar{U}=D_{n}, n>1$, by [7, Prop. 1], $\bar{U}$ is generated by the conjugates of $\bar{e}$, so that $\bar{U}=\bar{Q}$. The rest of the proof is unchanged.

(b) It is not hard to show that a subring (subalgebra) $B$ of a semisimple ring (algebra) $A$ is itself semisimple, if each homomorphism of $A$ which maps $A$ onto a primitive ring (algebra) also maps $B$ onto a primitive ring (algebra). 
(The proof of this is related to that of Corollary 2.2). Thus, if $A$ is a semisimple algebraic algebra, and if $P$ is any primitive ideal of $A$, then $[9$, p. 239 ff.] shows that the canonical map $A \rightarrow A-P$ maps $B=E(A)$ onto $E(A-P)$. If $A-P$ is not division, then $E(A-P)$ is primitive by (a), while if $A-P$ is division, since it is an algebraic division algebra, every nonzero subalgebra is a division algebra. Thus $E(A-P)$ is primitive in this case too, and the semisimplicity of $B$ follows from the remark above.

Relating to Lemma 5.2 is the question whether $T(A)=A$ in a simple ring (algebra) $A$ implies the equality $E(A)=A$.

\section{REFERENCES}

[1] Shimshon A. Amitsur, Invariant submodules of simple rings, Proc. Amer. Math. Soc. vol. 7 (1956), 987-989.

[2] Richard Arens and Irving Kaplansky, Topological representation of algebras, Trans, Amer. Math. Soc. vol. 63 (1948), 457-481.

[3] Michael P. Drazin, Rings with nil commutator ideals, Rend. Circ. Math. Palermo, vol. 6 (1957) 51-64.

[4] Carl Faith, Algebraic division ring extensions, Proc. Amer. Math. Soc. vol. 11 (1960), 43-53.

[5] Carl Faith, Radical extensions of rings, Proc. Amer. Math. Soc. vol. 12 (1961), $274-283$.

[6] Carl Faith, A structure theory for semialgebraic extensions of division algebras, J. für die reine und angewandte Math. (Crelle's Journal) vol. 209 (1962), 144-162.

[ 7 ] Carl Faith, Submodules of rings, Proc. Amer. Math. Soc. vol. 10 (1959), 596-606.

[8] Nathan Jacobson, Theory of Rings, Amer. Math. Soc. Surveys, vol. 2, Ann Arbor, 1948.

[9] Nathan Jacobson, Structure of Rings, Amer. Math. Soc. Colloquium Publications, vol. 37, Providence, 1956.

[10] Friedrich Kasch, Invariante Untermoduln des Endomomorphismenrings eines Vektorraums, Arch. der Math., vol. 4 (153), 182-190.

[11] Wallace S. Martindale, III, The structure of a special class of rings, Proc. Amer. Math. Soc. vol. 9 (1958), 714-721.

[12] Tadasi Nakayama, A remark on the commutativity of algebraic rings, Nagoya Math. J., vol. 12 (1959), 39-44.

[13] Yuzo Utumi, On ₹-rings, Proc. Japan Acad. vol. 33 (1957) 63-66.

[14] John von Neumann, On regular rings, Proc. Nat. Acad. Sci. (U.S.A.) vol. 22 (1936), $707-713$

Mathematisches Institut, Heidelberg, Germany

and

Institute for Advanced Study, Princeton, N. J. 